\title{
Characterization and Operation of a Liquid Crystal Adaptive Optics Phoropter
}

\author{
A. Awwal, B. Bauman, D. Gavel, S. S. Olivier, S. Jones, J. L. \\ Hardy, T. Barnes, J. S. Werner
}

This article was submitted to SPIE Annual Meeting 2003, Adaptive Optics Applications,

U.S. Department of Energy

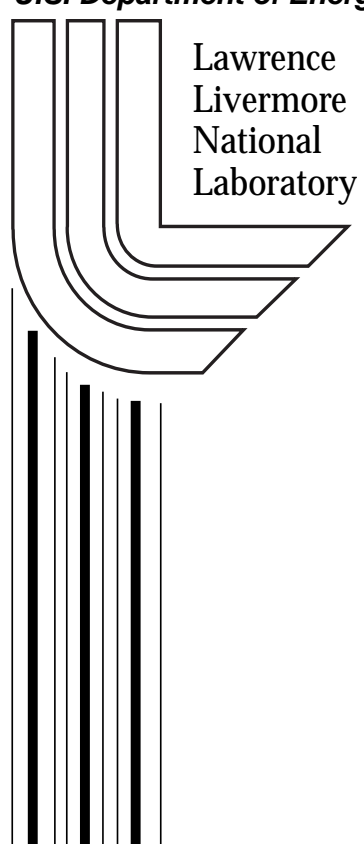

San Diego, California, August 3-9, 2003

February 5, 2003 


\section{DISCLAIMER}

This document was prepared as an account of work sponsored by an agency of the United States Government. Neither the United States Government nor the University of California nor any of their employees, makes any warranty, express or implied, or assumes any legal liability or responsibility for the accuracy, completeness, or usefulness of any information, apparatus, product, or process disclosed, or represents that its use would not infringe privately owned rights. Reference herein to any specific commercial product, process, or service by trade name, trademark, manufacturer, or otherwise, does not necessarily constitute or imply its endorsement, recommendation, or favoring by the United States Government or the University of California. The views and opinions of authors expressed herein do not necessarily state or reflect those of the United States Government or the University of California, and shall not be used for advertising or product endorsement purposes.

This is a preprint of a paper intended for publication in a journal or proceedings. Since changes may be made before publication, this preprint is made available with the understanding that it will not be cited or reproduced without the permission of the author.

This report has been reproduced directly from the best available copy.

Available electronically at http://www.doc.gov/bridge

Available for a processing fee to U.S. Department of Energy

And its contractors in paper from

U.S. Department of Energy

Office of Scientific and Technical Information

P.O. Box 62

Oak Ridge, TN 37831-0062

Telephone: (865) 576-8401

Facsimile: (865) 576-5728

E-mail: reports@adonis.osti.gov

Available for the sale to the public from

U.S. Department of Commerce

National Technical Information Service

5285 Port Royal Road

Springfield, VA 22161

Telephone: (800) 553-6847

Facsimile: (703) 605-6900

E-mail: orders@ntis.fedworld.gov

Online ordering: http://www.ntis.gov/ordering.htm

OR

Lawrence Livermore National Laboratory

Technical Information Department's Digital Library

http://www.llnl.gov/tid/Library.html 


\title{
Characterization and Operation of a Liquid Crystal Adaptive Optics Phoropter
}

\author{
Abdul Awwal*, Brian Baumann*, Don Gavel*, Scot Olivier*, Steve Jones*, Joseph L. \\ Hardy $\$$, Thomas Barnest and John S. Wernert \\ *Lawrence Livermore National Laboratory, Livermore, CA 94550 \\ $\$ U C$-Davis Medical Center, Department of Ophthalmology, Sacramento, CA 95816
}

\begin{abstract}
Adaptive optics (AO), a mature technology developed for astronomy to compensate for the effects of atmospheric turbulence, can also be used to correct the aberrations of the eye. The classic phoropter is used by ophthalmologists and optometrists to estimate and correct the lower-order aberrations of the eye, defocus and astigmatism, in order to derive a vision correction prescription for their patients. An adaptive optics phoropter measures and corrects the aberrations in the human eye using adaptive optics techniques, which are capable of dealing with both the standard low-order aberrations and higherorder aberrations, including coma and spherical aberration. High-order aberrations have been shown to degrade visual performance for clinical subjects in initial investigations. An adaptive optics phoropter has been designed and constructed based on a ShackHartmann sensor to measure the aberrations of the eye, and a liquid crystal spatial light modulator to compensate for them. This system should produce near diffraction-limited optical image quality at the retina, which will enable investigation of the psychophysical limits of human vision. This paper describes the characterization and operation of the AO phoropter with results from human subject testing.
\end{abstract}

Keywords: Adaptive Optics, Vision Science, liquid crystal, spatial light modulator, ophthalmology, psychophysical testing, visual acuity,

\section{INTRODUCTION}

A normal eye suffers from aberrations of the lens and cornea, which blur both our vision of the world and also and also interfere with ophthalmoscopic micro-visualization of our retina. Corrective lenses can generally improve vision to 20/20 Snellen acuity, but these only correct very low-order aberrations, defocus and astigmatism. The high-order aberrations remains untreated, and can continue to affect visual performance. In addition, low-order correction is not enough to reveal a clear microscopic structures in the retina, which could be used improving diagnosis of retinal diseases that cause loss of vision, such as age-related macular degeneration, and also for in vivo monitoring the effects of newly developed drug treatments in the human eye.

Adaptive Optics (AO) has been used successfully for astronomical applications for over a decade. In this technique, the atmospheric aberration is measured and then corrected by 
applying the inverse aberration to the light collected by the telescope using a deformable mirror. Extending the AO technology to vision applications can lead to correction of high-order aberrations in the eye and produce better than 20/20, or supernormal vision. In addition, it is possible to produce very high fidelity retinal images. LLNL first demonstrated the use of adaptive optics technology using a sodium laser guide star for astronomical imaging [1]. Using the same type of Shack-Hartmann sensor that was used in astronomical adaptive optics [2,3], Liang et al. demonstrated a new technique for measuring the aberration of the eye [4]. Subsequently, at the University of Rochester, Liang, Williams and Miller [5] added a wavefront correction to the wavefront measuring system and demonstrated the first application of adaptive optics to improve human vision. They also made the first demonstration of the correction of the high-order aberrations to obtain never-before-seen images of the living human retina [5].

The vision correction technology used in the Rochester AO system [5] is a conventional deformable mirror device (DMD) as used in astronomical applications. In addition to being expensive, DMDs have much larger apertures than the eye. This leads to a large optical system in order to allow for magnification of the $8 \mathrm{~mm}$ pupil of the eye to the larger size of the deformable mirror. This combination of cost and size limits the suitablility of an AO system using a DMD for clinical trials and eventual commercialization.

Recently new AO technologies have been developed based on both liquid crystal (LC) devices and micro-electro-mechanical mirrors, which are both compact and less expensive than the conventional DM devices. The AO group at LLNL has previously demonstrated very high order wavefront correction using LC technology [6]. One of the purposes of this work is to test these new technologies for vision correction. This work laso exploits the experience of LLNL AO group in astronomical imaging with AO [7].

This paper presents the design [7] and characterization and application of an adaptive optics phoropter based on LC technology. The paper is organized into four sections. The first section describes the optical setup and hardware. The second section describes the calibration of the optical components, and the third section elaborates on the closed-loop operation and performance verification of the phoropter. The fourth section describes the operation with human subjects. 


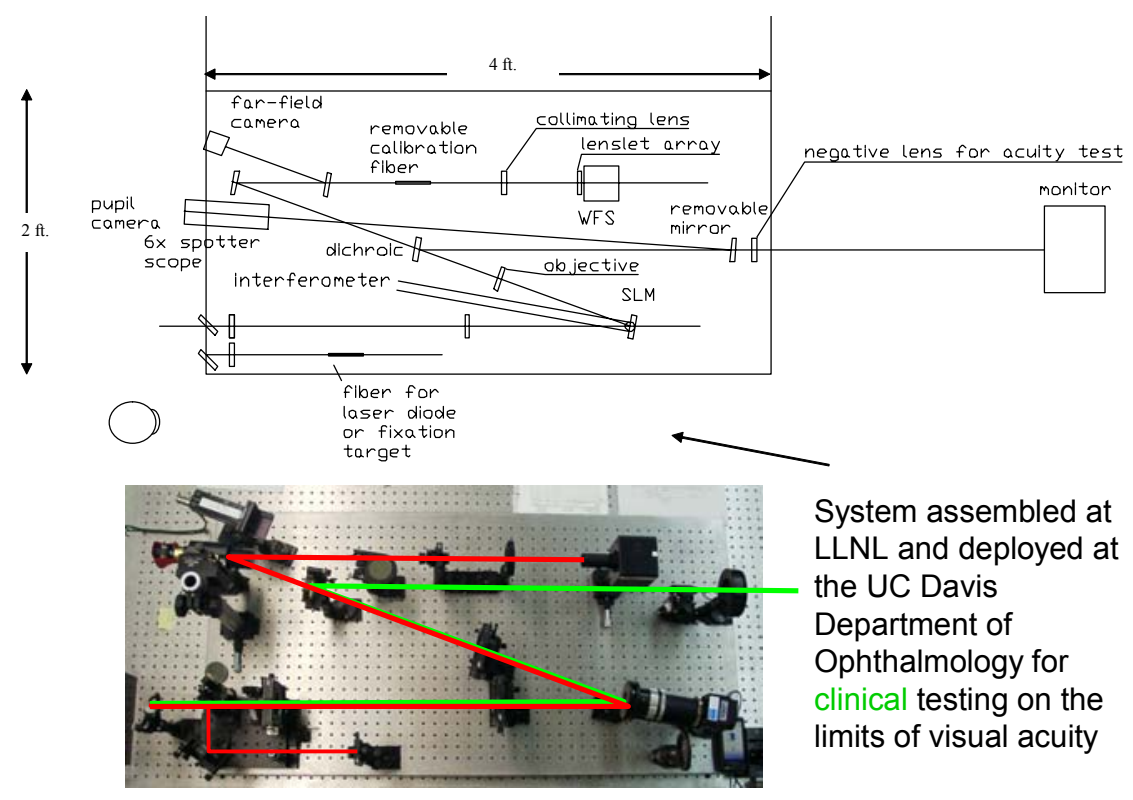

Fig. 1 Prototype adaptive phoropter using liquid crystal spatial light modulator

\section{OPTICAL SET UP AND HARDWARE}

A schematic of the high resolution adaptive optics phoropter system is shown in Fig. 1. The instrument uses a 5 microwatt laser at $785 \mathrm{~nm}$ that is focused onto the retina of a human eye. The laser beacon is reflected off the back of the retina and out through the optics of the eye, thereby sampling their aberrations. The light reflected $(\sim 0.02 \%)$ by the retina is transmitted to a Shack-Hartmann (S-H) wavefront sensor after being reflected by a wavefront corrector. This arrangement allows for a closed-loop correction of the optical aberration. Here, the S-H sensor measures the wavefront after it is falttened by the wavefront corrector. The deviation of the reconstructed wavefront from an ideal flat wavefront is estimated, and this produces the error term. This error term is used to calculate the correction required to compensate for the deviation.

A control loop is used to update the correction applied to the wavefront corrector in a stable fashion. During the closed-loop operation of the AO system, a correction is applied until the error reaches a minimum possible value. When the correction is applied through the closed-loop system for the aberration from an eye, enhanced vision will result. After the system has converged to a stable low aberration value, the subject is asked to look at a computer monitor displaying some selected patterns. Thereafter, psychophysical tests are carried out with the system to understand the effect of high order correction on the visual performance. Figure 1 illustrates two light path. The red beam shows light emerging from the eye and going into the wavefront sensor after being reflected by the wavefront corrector. The green beam represents rays from the visual acuity target traveling to the subject's eye. 
Next the two major subsystems are described as well as their calibration procedures required for meaningful operation can be performed.

The wavefront corrector SLM: The spatial light modulator, which can control approximately 230,000 phase control points, serves as the wavefront corrector in the proposed AO phoropter. The parallel aligned nematic liquid crystal spatial light modulator (PAL-SLM) is an optically addressable (intensity to phase) spatial light modulator, as shown in Fig. 2. The PAL-SLM has an amorphous silicon layer, a dielectric mirror, and liquid crystal layer sandwiched between two glass substrates with transparent electrodes. A write light beam impinges on the amorphous silicon side, and the read beam is presented on the LC side. The impedance of amorphous silicon becomes extremely high when no write light is present. When the write light is applied, the impedance is lowered, and a voltage applied across the liquid crystal layer increases depending on the intensity of the write beam. The increase in voltage across the liquid crystal layer affects the molecular orientation and changes the index of refraction causing a phase modulation of the read beam.

To control the optical intensity of the write beam, a laser diode is coupled with a liquid crystal display (LCD) used in transmissive mode. This allows projection of any intensity pattern on the write side of the PAL SLM. The PAL SLM module (PAL SLMM) combines the laser diode and LCD combination with the PAL-SLM so that the entire system as a whole acts as an electronically addressable phase/intensity spatial light modulator. The SLM contains individually addressable $480 \times 480$ control points with a 0.8 micron stroke on a $20 \mathrm{~mm} \times 20 \mathrm{~mm}$ surface.

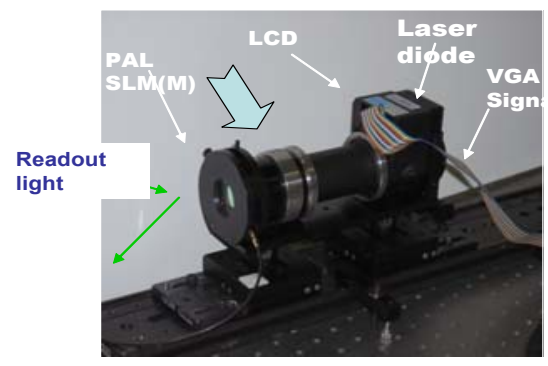

Fig. 2. The liquid crystal SLM Module

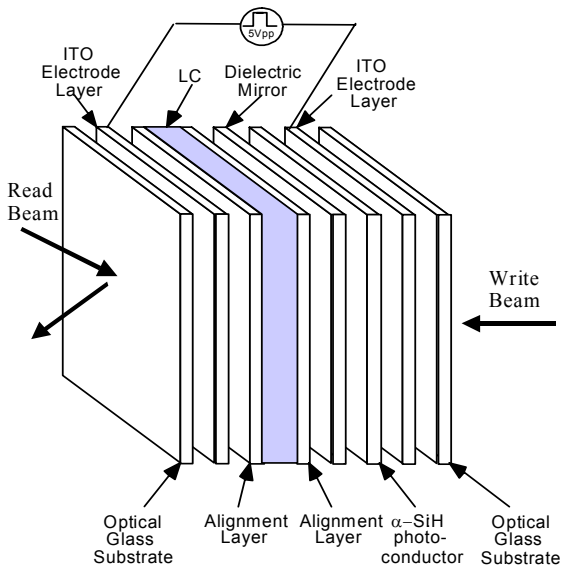

The wavefront sensor: Shack-Hartmann sensor: The Shack-Hartmann sensor (Hartmann sensor modified by Shack) serves as the wavefront aberration measuring device. It consists of an 8-bit digital camera coupled with a lenslet array. When a plane 
wave is incident upon the sensor, it produces a regular array of spots, which are located on the optical axis of the corresponding lenslet. This is taken as reference pattern. If the wavefront has a phase distortion, the focal spot of each subaperture shifts with respect to corresponding point on the reference pattern by a factor proportional to the local tilt. The position of the focal spot is determined by the centroid operation. The local slopes are related to the shift in the centroid positions. This relationship can be estimated from the dimensions of the S-H sensor. The lenslet diameters are 203 microns with a focal length of $5.8 \mathrm{~mm}$. The $\mathrm{S}-\mathrm{H}$ sensor has $20 \times 20$ subapertures. The camera pixels are 16 microns square. The scale of the $\mathrm{S}-\mathrm{H}$ sensor is $16 / 5.8=2.75 \mathrm{mrad} /$ pixel. Thus a centroid will shift by one pixel for a local tilt at a subaperture of $2.75 \mathrm{mrad}$. This is equivalent to a 0.56 micron phase jump between two subapertures.

It is possible to estimate the camera response given a certain amount of expected power. From the camera characteristics curve it is evident that the camera produces 10 digital numbers (DN) for $1 \mathrm{~nJ} / \mathrm{cm}^{2}$ intensity. This is equivalent to 1011 photons/pixel. For $5 \mu \mathrm{W}$ power incident on the retina, $0.02 \%$ is reflected. This $1 \mathrm{nW}$ power incident on $20 \times 20$ subapertures for $100 \mathrm{~ms}$ produces about $195 \mathrm{DN}$, and is enough for detection of the wavefront.

- Angle subtended by one pixel $=(\mathrm{dx} / \mathrm{f})=16 \mathrm{mi} / 5.8 \mathrm{~mm}=$ $2.75 \mathrm{mrad}$

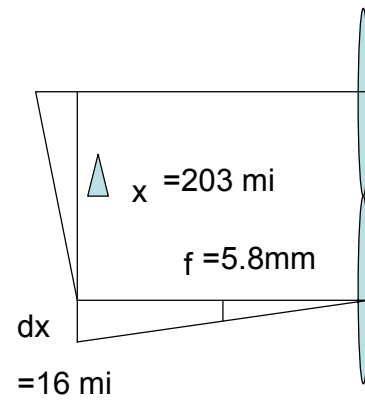

Lift/sub-ap $=$ Angle ${ }^{*}$ delta $(x)$

Lift/sub-ap/pixel move $=$ $=(16 \mathrm{micron} / 5.8 \mathrm{~mm})^{\star} 203 \mathrm{mi}$

$=0.56$ microns

standard deviation of the centroid with time using center of mass

$=0.04-0.05$ pixels $\sim 0.03$ microns $\sim(1 / 25)$ th waves at $785 \mathrm{~nm}$

for Gaussian fit $=0.0047 \sim(1 / 300)$ waves

Fig. 3 The measurement of temporal variations

Shack Hartmann Noise: The noise statistics of the camera were measured for various camera integration times. The statistics provides us with the noise mean level (or dark noise) as well as the variance of the noise level, which determines the minimum detectable noise power. For example, for an integration time of $110 \mathrm{~ms}$, the noise floor is at 20.7 pixel count, while the standard deviation of the noise at any pixel is at 0.03 counts for dark exposure. When uniform light is exposed, the centroid rms for 100 frames calculated using center of mass algorithm with dark subtract is 0.04 to 0.05 pixels. 
Nonlinear Characterization of the SLM response: A set of experiments was carried out to determine the phase modulation property of the liquid crystal SLM. This will provide the grey level needed to achieve a certain value of phase modulation.

The whole SLM was characterized by applying a periodic rectangular wave of varying amplitude to the SLM and measuring the far field pattern. The relative magnitude of the $0^{\text {th }}$ order and the first order components gives us an indication of the phase jump magnitude. For example, for a phase jump of $\pi$, the 0th order intensity becomes zero, while the first order intensity becomes maximum. The same phenomenon is observed across each of the subapertures of the S-H sensor which instead of a big lens has a microlens array. Thus, a second space variant test was developed to determine the phase response of individual actuators. If a step function of varying amplitude is applied over a single aperture, each subaperture produces a farfield pattern corresponding to the Fourier transform of the phase jump. The history of all the wavefront sensor responses as a function of the amplitude of the step function was recorded. The phase response of individual actuators was calculated. A look up table for the SLM was devised, combining the individual response with the overall response. The desired phase angle is the input to the lookup table and the required grey level of the SLM to cause that phase change is the output.

The phase-response behaviors obtained from the individual subapertures reveal that the SLM has space-variant phase modulation characteristics. A spatially varying lookup table was devised which compensated for the nonuniformity of the phase response across the SLM surface. The phase response from the lookup table is expressed in the curve shown in Fig. 4. The x-axis represents the phase angle (in radians multiplied by 40) while the y-axis represents the driving grey level for the SLM.

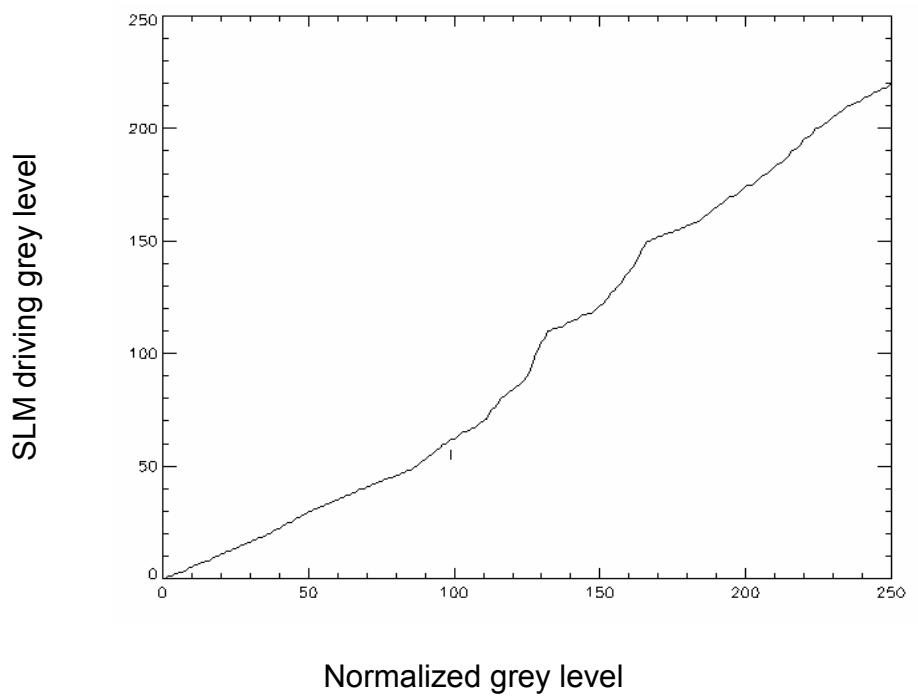

Fig. 4 The look up table for the phase response of the SLM 
Phase Wrapping: This curve can be used to perform phase wrapping. In order to verify the phase wrapping, a Gaussian input varying from 1 to 3 waves was applied to the SLM. The corresponding reconstructed wavefront was observed. The peak value of the input and output wavefront was recorded. The plot of the input versus output is shown in Fig. 5.

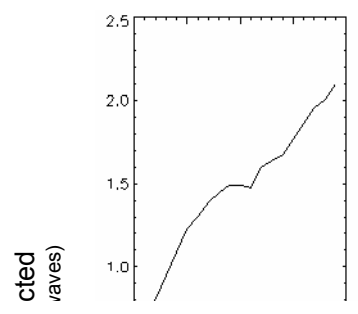

Registration: A systematic procedure was developed-to register the SLM on the wavefront sensor. If the SLM is not properly registered with the wavefront sensor, then correction calculated from the wavefront measurement will be misaligned with respect to the position of the aberration on the incoming wavefront. Figure 6 illustrates an example of this problem. An initial aberration is applied to the SLM and this elicits acompnesating correction. Due to misregistration, the measured wavefront sensor sees the aberration in one location but the AO system applies the correction at a different location. 


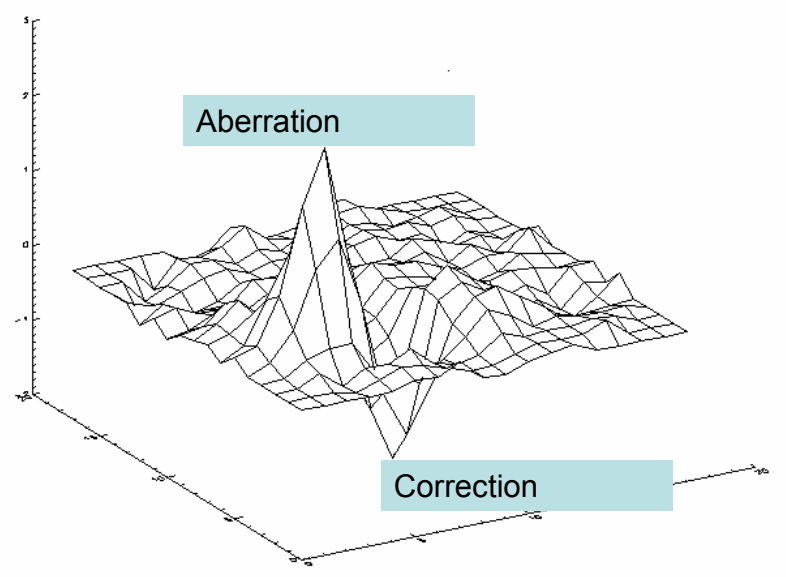

Fig. 6 Effect of mis-registration: correction applied at a different place

This position error contributes to the next iteration through the closed loop, resulting in a new incorrect position for the wavefront correction. Fig. 7 shows the results after 3,5 and 11 iterations. A misregistration of the SLM in the x-direction appears as vertical lines evolved on the SLM caused by a ripple effect of the misplaced correction.

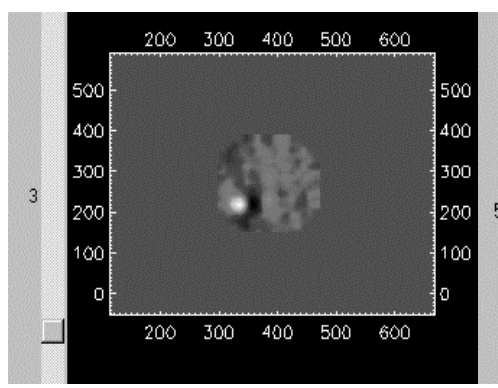

After 3 iterations

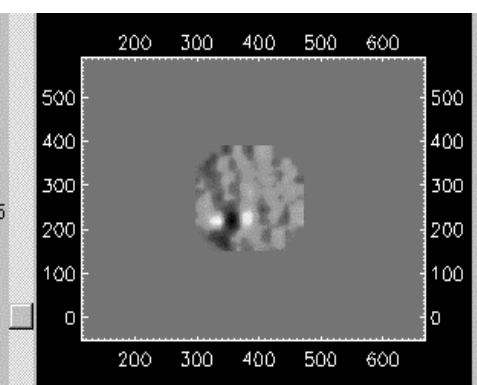

After 5 iterations

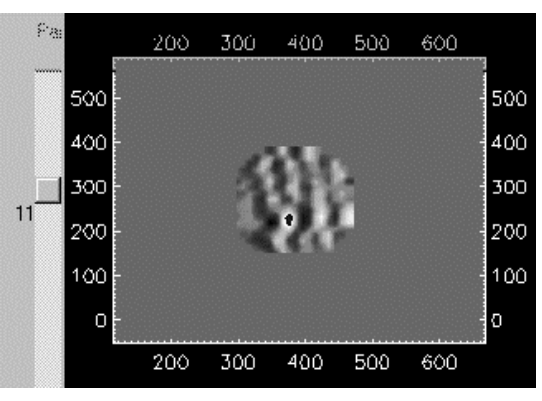

After 11 iterations

Fig. 7 Effect of misregistration 


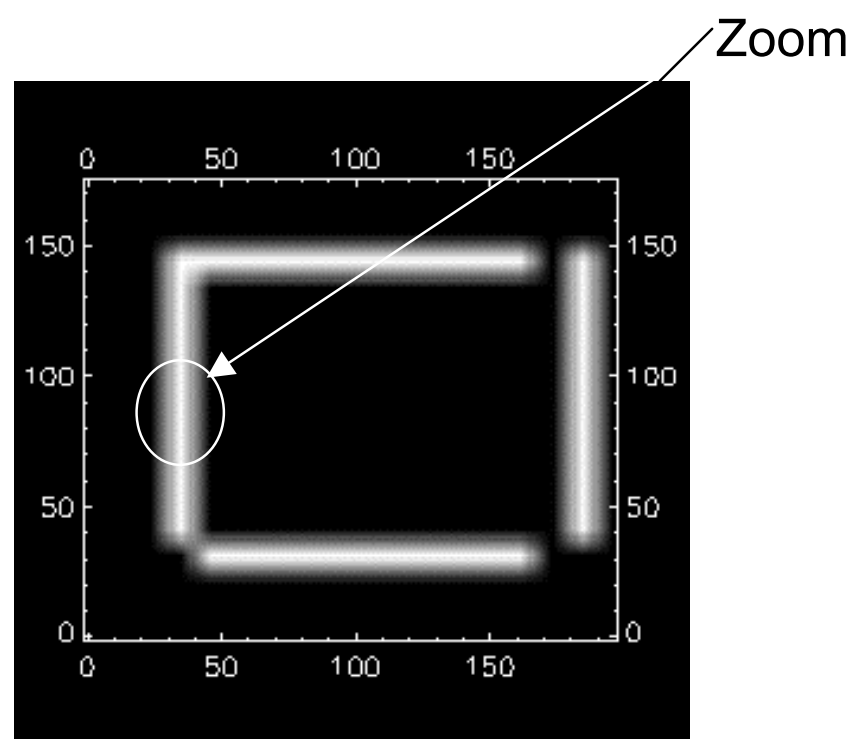

Input on SLM

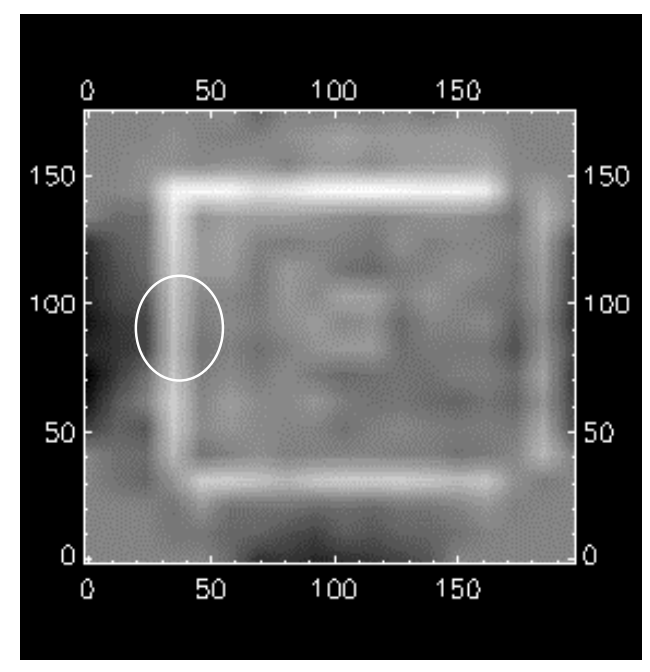

Reconstructed output

\section{Fig. 8 Registration: experiment}

In this method, an asymmetric pattern of known size and shape was applied on the SLM. The actuation function is compared to the wavefront reconstructed obtained from the wavefront sensor data. Making this comparison provides information about the registration in terms of rotation, scale and position of the SLM with respect to the
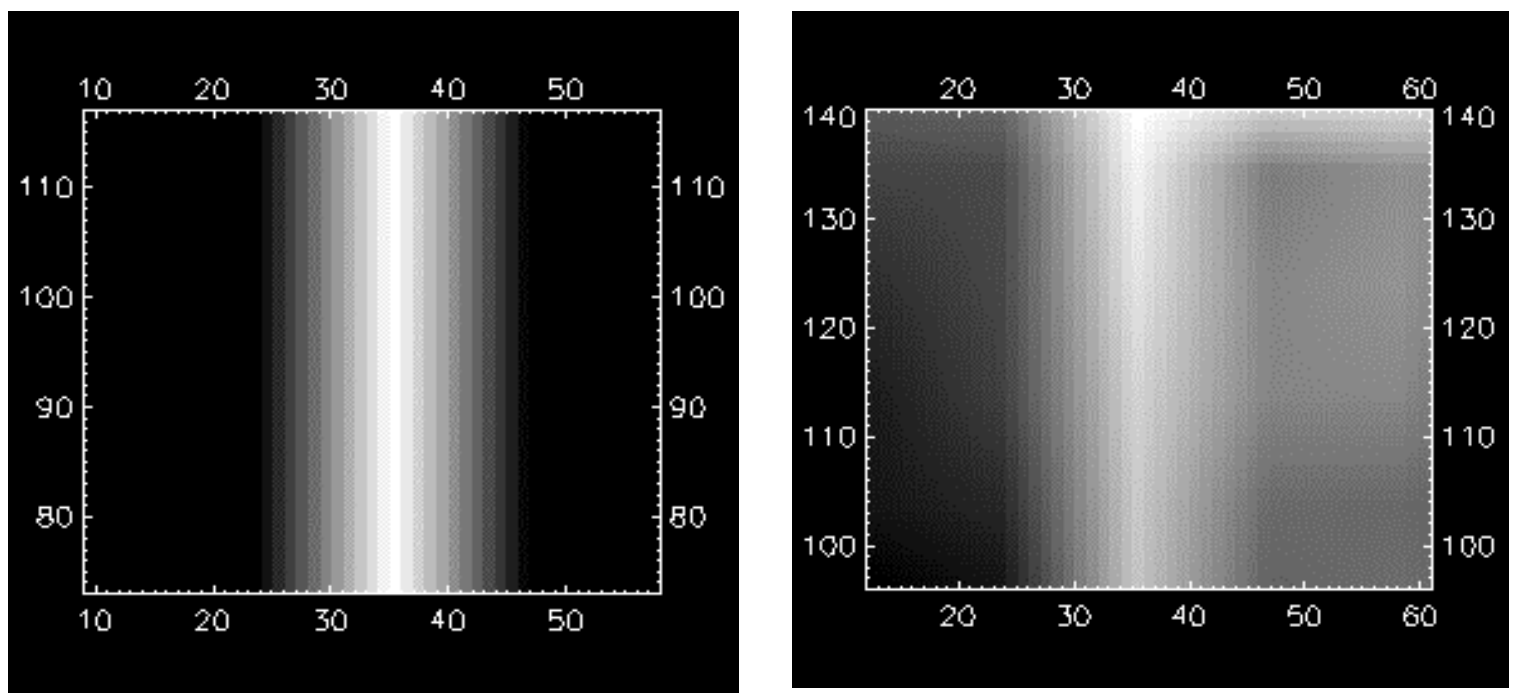

Fig. 9 Finding $x$ correspondence (a) written pattern on SLM (b) detected pattern on wave front sensor

wavefront sensor. For improved accuracy, the comparison was performed at the pixel levels of the SLM plane. Having compared the actuation function and the reconstructed 
wavefront, the size and position of the pattern is modified until the written and detected pattern correspond. The process yields four parameters, the size of the pattern (two parameters) and its position ( $\mathrm{x}$ and $\mathrm{y}$ position). These four parameters describe exactly the position of the written pattern on the SLM.

\section{Biased Operation of SLM}

A typical correction pattern reveals that both positive and negative corrections must be applied to compensate an aberration. This is achieved by operating the SLM at a $\pi$ bias. This allows both positive and negative corrections to be applied. As a result of the bias point, the first phase wrap occurs after a $\lambda / 2$ wave excursions; the second phase wrap will occur after $3 \lambda / 2$.

\section{CLOSED LOOP OPERATION}

In order to verify the proper operation of all the subsystems, the AO system must be operated in a closed loop. In the closed loop mode, the system is operated to minimize the error in the wavefront from a perfect flat. To test the system, an artificial aberration is applied through the SLM. Since the wave front sensor will register the function as an aberrated wavefront, the control loop will work by reducing the error. If the system is working properly, as shown in the following figure, the wavefront error gradually reduces.

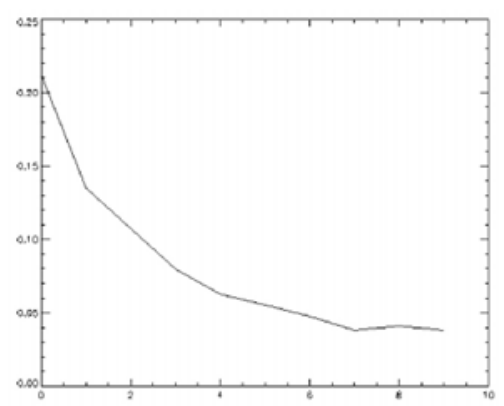

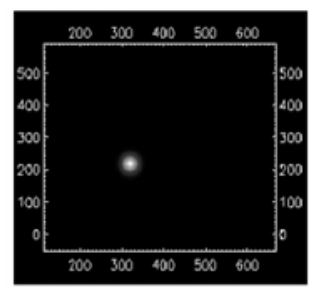

iteration 0

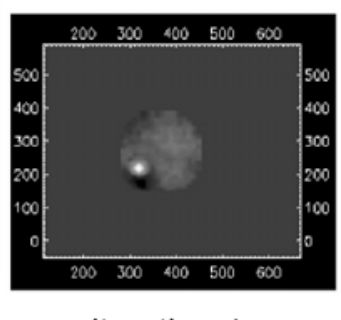

iteration 4

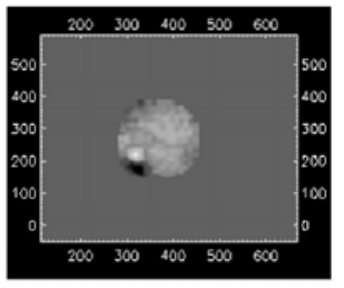

iteration 8 iteration number

Fig. 10 Convergence of a Gaussian actuation

Wavefront reconstruction: The wavefront is calculated by comparing its deviation from a plain wave. To determine the plane wave position, the Shack-Hartmann sensor is illuminated with a plane wavefront. In each subaperture the light will be focused at the optical axis of that subaperture. This position is marked by calculating the centroid of the illuminated region. Thus these are called the reference centroids.

When there is a phase gradient across the wavefront, the light will be tilted and the focus point at each subaperture will be shifted by a proportional amount. When an aberrated wavefront is sampled by the S-H sensor, the locations of the new centroids are shifted according to the local phase gradient. These new centroids are calculated, and the difference of these centroids from the previous position provides a measure of the local 
slope of the wavefront $\frac{d \phi}{d x}=\frac{d}{f}$ where $\mathrm{d} \varphi / \mathrm{dx}$ is the slope of the wavefront and $d$ is the displacement of the centroids. A set of discrete linear equations can be written relating the slopes to the derivative and eventually to the displacement of the centroids. This leads to a discrete set of equations relating the phase to the slope. These equations can be solved by either a least squares method or Fourier transform method to yield the reconstructed wavefront. In our system, we utilize a Fourier transform technique to reconstruct wavefront from the centroid differences [8].

\section{Algorithm}

The algorithm for closed loop error corrections consists of the following steps:

1. Retrieve reference centroid from a plane wavefront.

2. Get new centroids from the aberrated wavefront.

3. Threshold both image for noise and dark noise.

4. Calculate centroids and hence the difference of both centroids

5. Input the difference centroids data to the reconstructor and construct the wavefront.

6. Estimate the correction applied per iteration which is a function of the gain

7. Convert the correction (in phase units) to the SLM units using a look-up table or simple formula.

8. Repeat steps 2 to $7 \mathrm{~N}$ times or until the error is below a certain threshold value.

Centroid calculation: Accurate calculation of the centroid is one of the most important fundamental operations in wavefront reconstruction. The variance of the various centroid calculation algorithms such as center of mass, Gaussian fitting, diminishing area of interest, was compared. The standard deviation of the center of mass are of the order of $10^{-2}$ where as those due to Gaussian fitting were on the order of $10^{-3}$. For good Hartmann spots, the Gaussian method did provide excellent repeatability in the presence of noise, however, for the real eye this method tends to fail because of shift of the centroids from their reference position and low light levels.

The centroids are calculated using a center of mass algorithm and a Gaussian fit. It should be noted that a typical subaperture after a dark subtract shows a significant amount of 
background noise around the central peak as shown in Fig. 11. One of the problems with

$\begin{array}{rcrrrrr}0 & 1 & 2 & 19 & 11 & 4 & 0 \\ 1 & 3 & 11 & 48 & 21 & 5 & 0 \\ 2 & 19 & 49 & 226 & 107 & 34 & 7 \\ 1 & 7 & 17 & 80 & 35 & 18 & 2 \\ 0 & 3 & 3 & 19 & 18 & 6 & 0 \\ 0 & 0 & 1 & 4 & 3 & 0 & 0 \\ 0 & 0 & 0 & 3 & 3 & 0 & 0\end{array}$

Fig. 11 A typical subaperture after subtracting dark image, note the presence of noise outside the main lobe 1.7 pixels

finding an appropriate threshold to exclude the background is the variance of intensities among different subapertures when a human eye is used. The pyramidal thresholding seems to do a better job in reducing the noise. An rms measurement reveals the variation of centroids over time; however, it may not reveal a systematic error present in any subaperture due to presence of excess noise or noise peak. To visually compare the performance of various centroiding algorithms a reconstructed wavefront is shown in Fig. 12 and 13. Here the rms wavefront error is calculated over only the non-central peak value. These values should ideally be zero but they show a non-zero value. In addition, a curve is plotted to show the noise versus the width of the apertures. Other systematic error sources that may creep up are in the floating point calculation of differences centroids. 


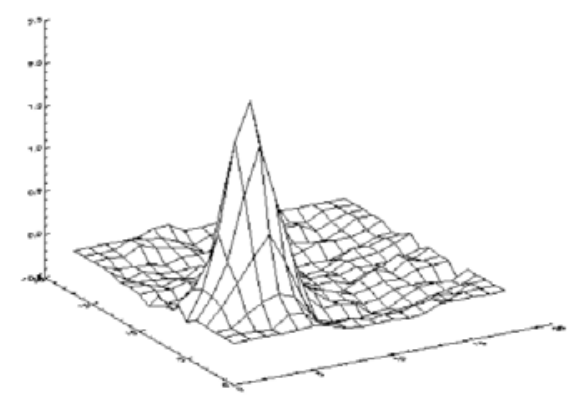

Subaperture size $=11$

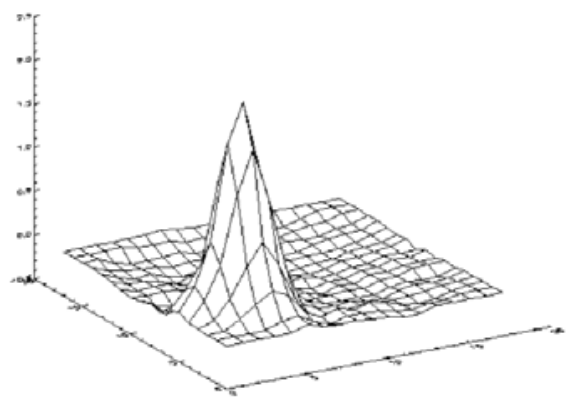

Subaperture size $=7$

Fig. 12 Pyramidal centroiding

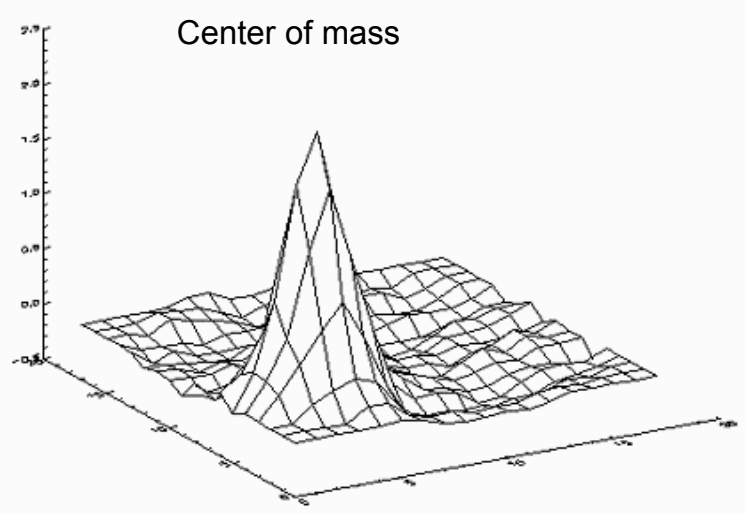

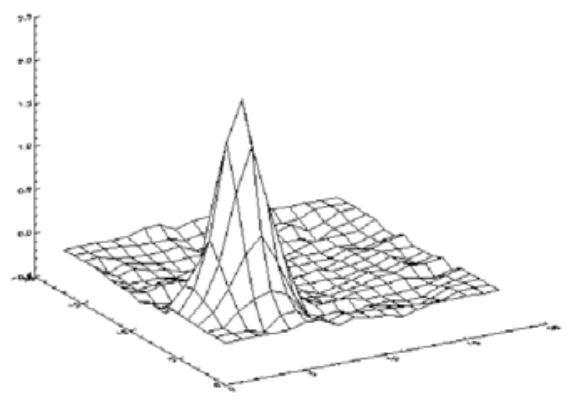

Subaperture size $=9$

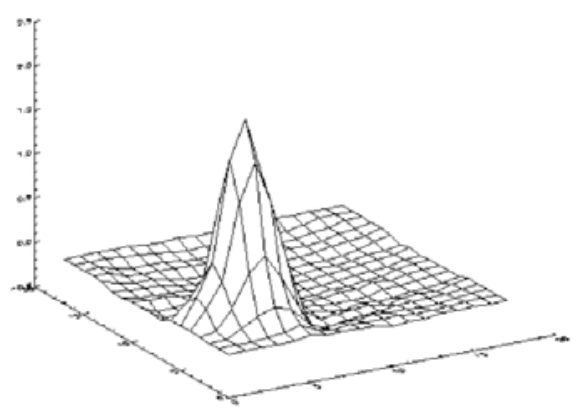

Subaperture size $=5$

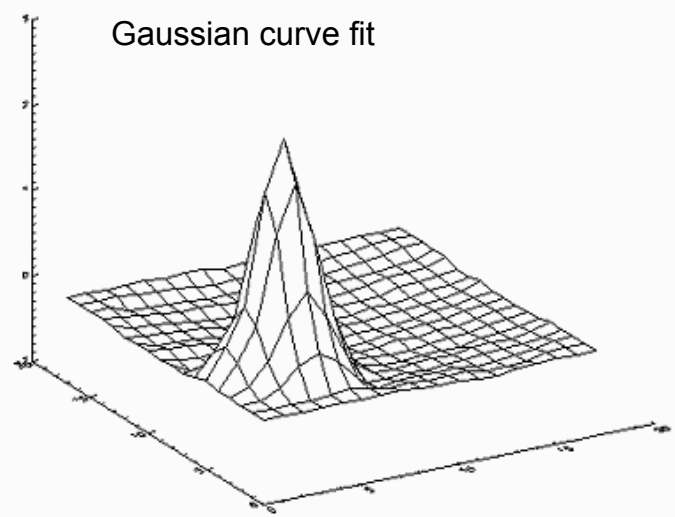

Fig. 13 Comparison of centroiding

\section{Measuring wavefront: verification}

First, an open loop measurement is performed with a known object in the system such as a lens of a certain focal length. For this experiment, a mechanical eye consisting of a rotating disk at the position of the human eye is used as a reference source. The rotation reduces the speckle in the Hartmann spots. A 0.25 diopter lens is placed on the phoropter 
which is physically located in front of the mechanical eye source. The wavefront is measured and verified to be exactly 2 microns.

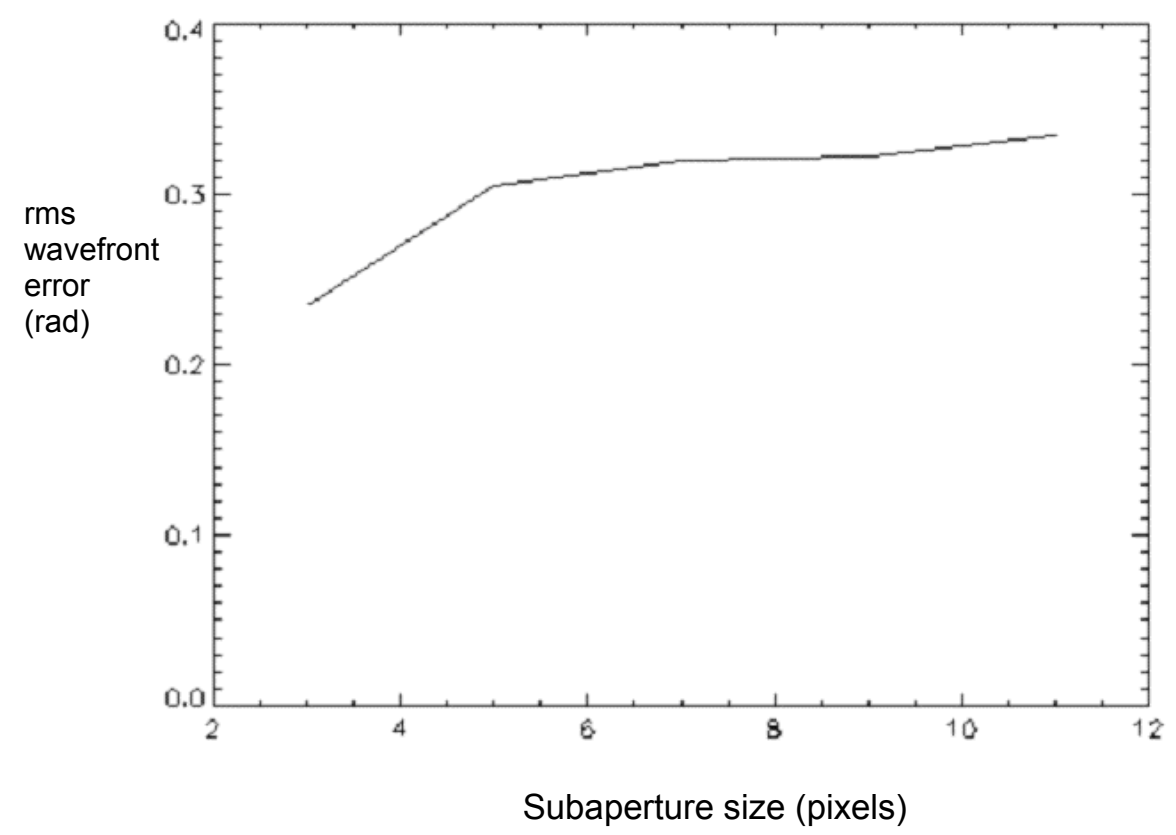

Fig. 14 Effect of subaperture size

To verify the closed loop operation, an artificial aberration is applied on the SLM. This is shown in Fig. 16. Then the loop is closed on this aberration. As shown in Figure 17, the aberration is gradually compensated by the correction applied through the control loop. The rms error of wavefront reduces from 0.34 radians to 0.24 radians. 


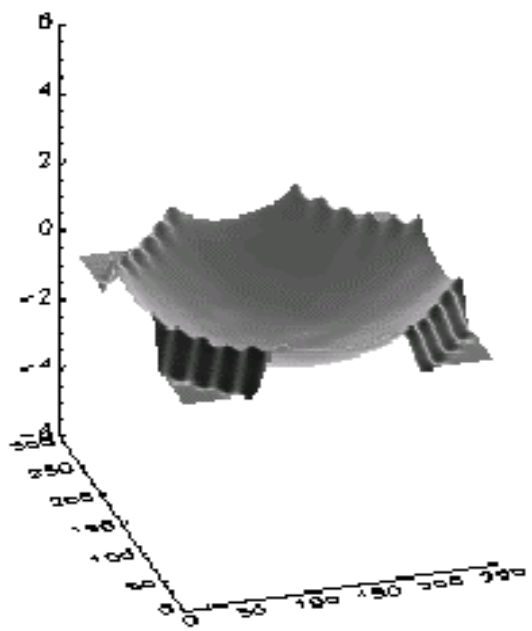

Fig. 15. Measurement of a 2 microns lens
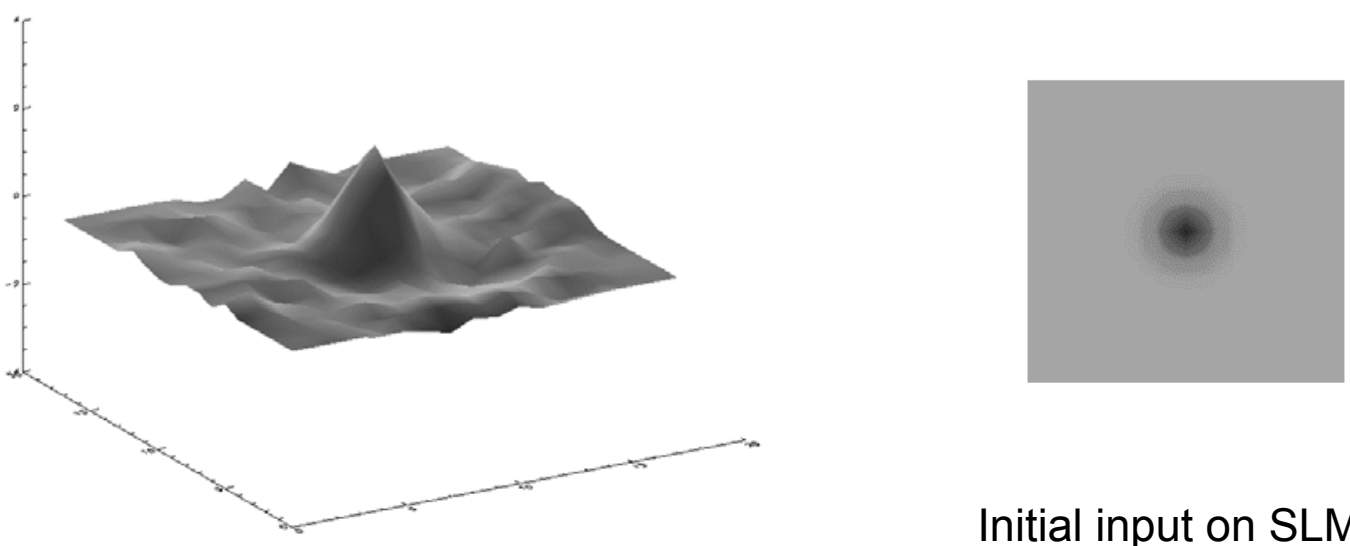

Initial input on SLM

Reconstructed phase

Fig. 16 An aberration input on the SLM 


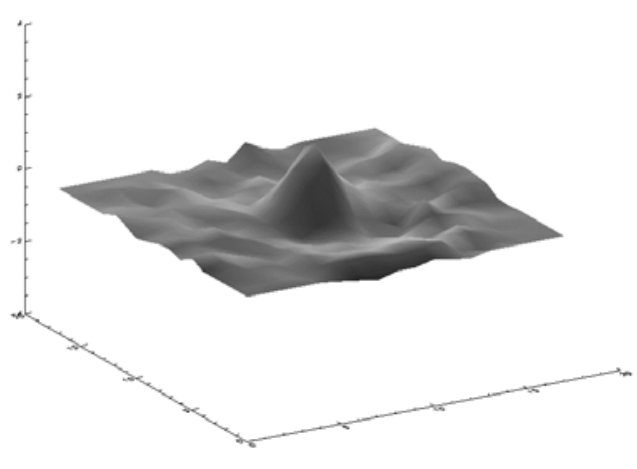

$2^{\text {nd }}$ iteration

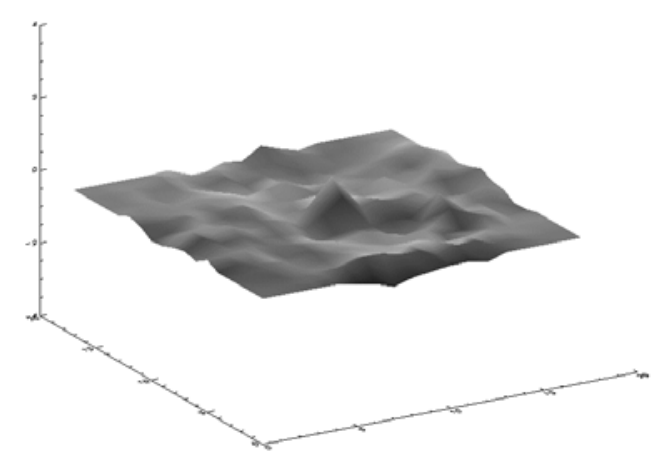

$6^{\text {th }}$ iteration

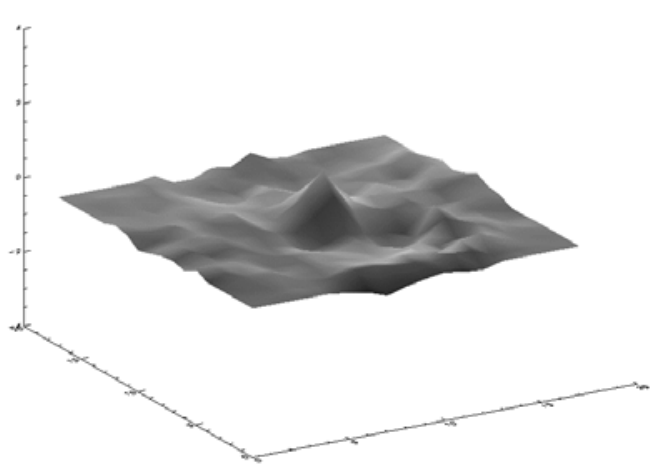

$4^{\text {th }}$ iteration

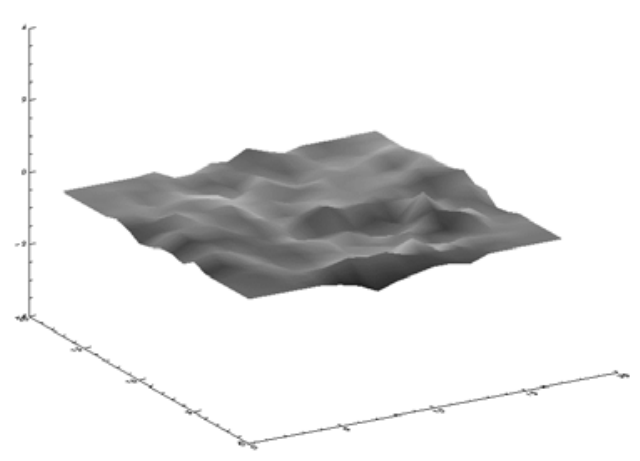

$10^{\text {th }}$ iteration

Fig. 17. Wavefront (in radians of phase) after 2,4,6,10

Next an external aberration is applied to the system using a phoropter lens. The total aberration produced by this is $0.25 \mathrm{D}$ lens is 2 microns or $\sim 3$ waves. Thus to compensate this aberration using the SLM which has about 0.8 micron stroke, phase wrapping must be performed to extend the range of the compensation to multiple waves. Figures 19 and 20 show the wavefront flattened by the AO control.

Fig. 21 shows the SLM actuations after $2^{\text {nd }}$ and $24^{\text {th }}$ iterations. The second panel of Fig. 18 demonstrates the wrapping of the phase at $0.5,1.5$ and 2.5 waves above the 0.5 bias level, which is necessary to correct an aberration of 3 waves. The grey background shows a constant bias of 0.5 waves. As shown in Fig. 22, the error is gradually reduced from 0.7 microns rms to 0.05 . 


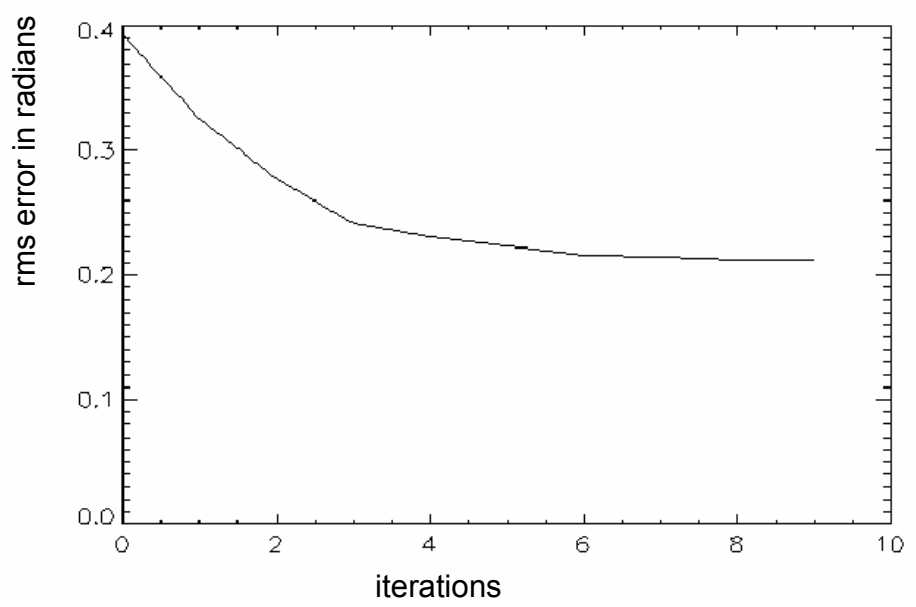

Fig. 18. Convergence of the internal aberrations to 0.024 microns

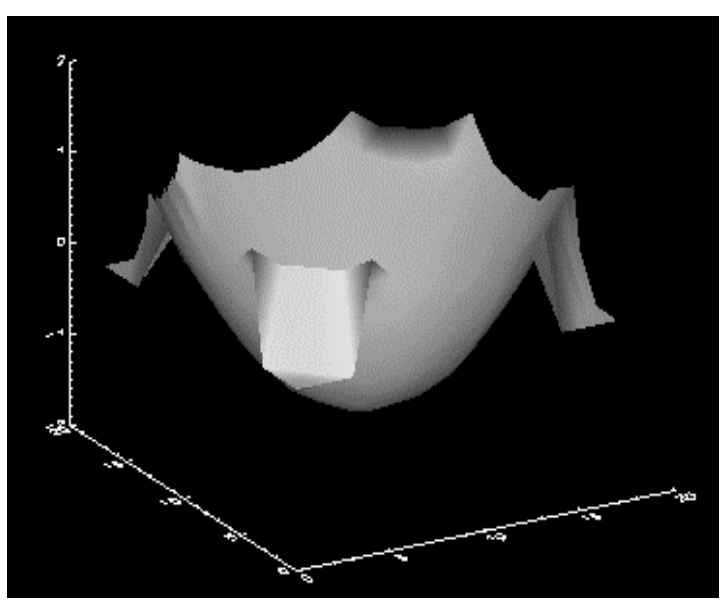

$0^{\text {th }}$ iteration

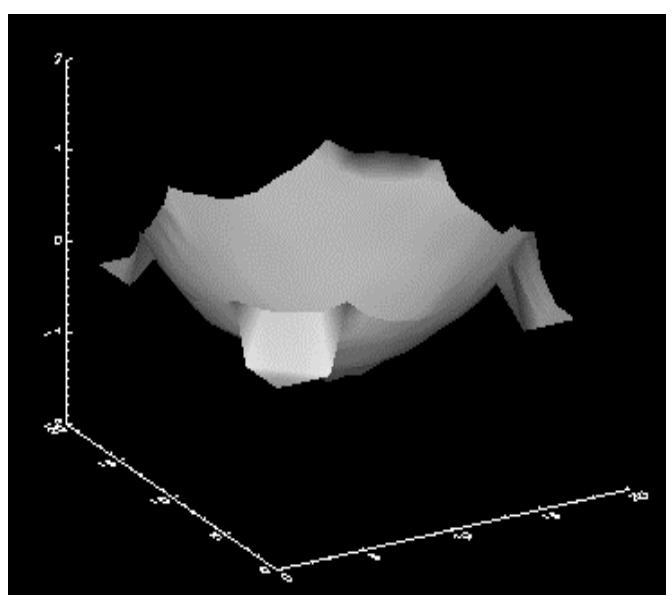

3rd iteration

Fig. 19 a .5 diopter lens in the system initial aberration
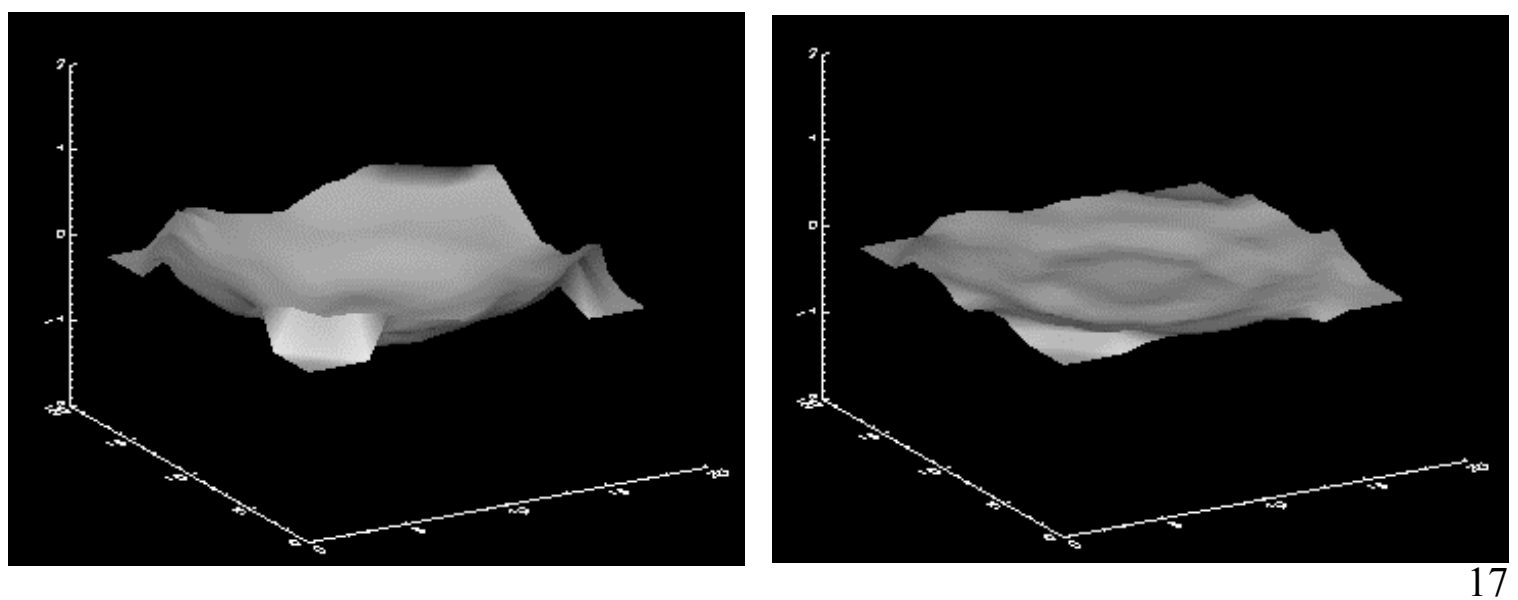

Fig. 20. Convergence of a focus aberration: 5 th and 9th iterations 

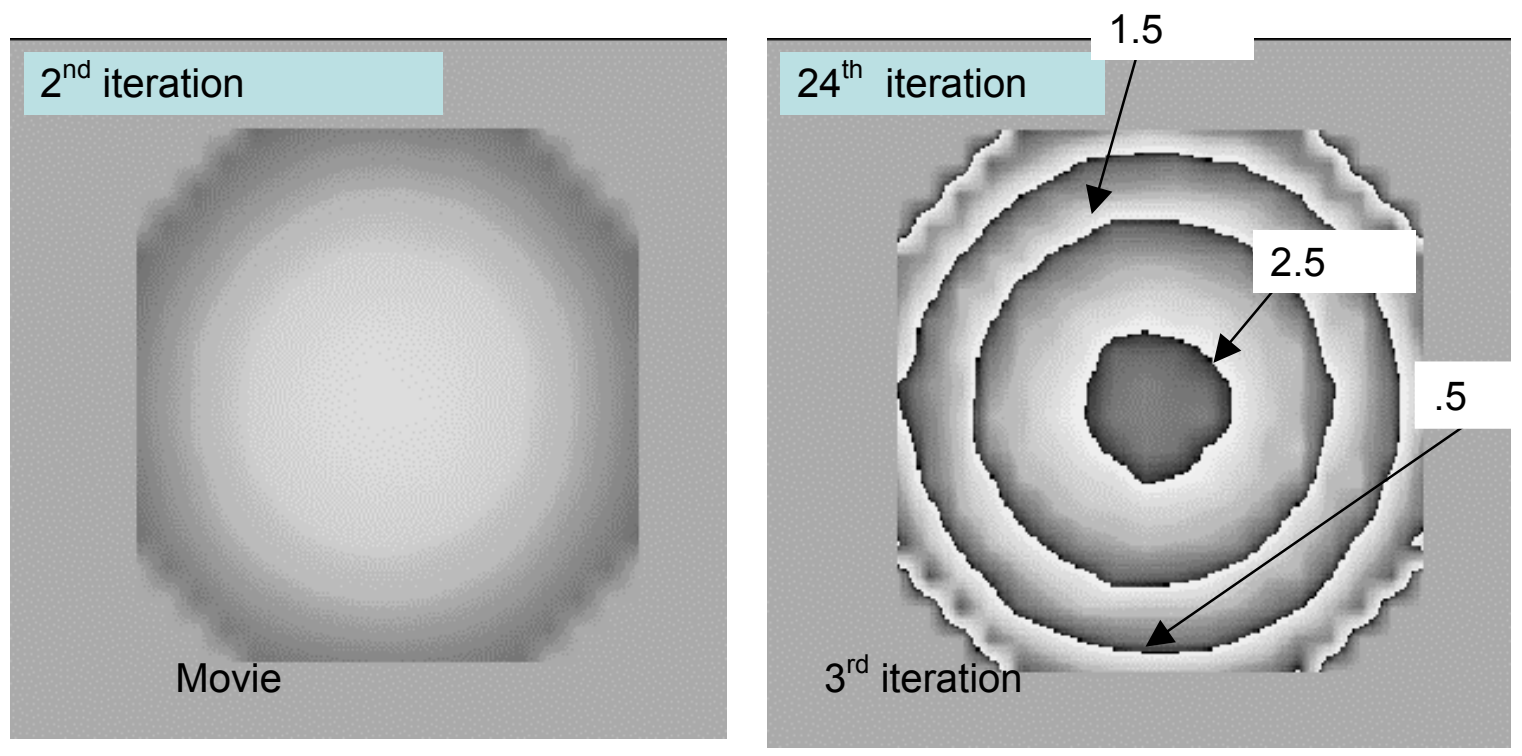

Fig. 21. SLM actuations to correct spherical aberration

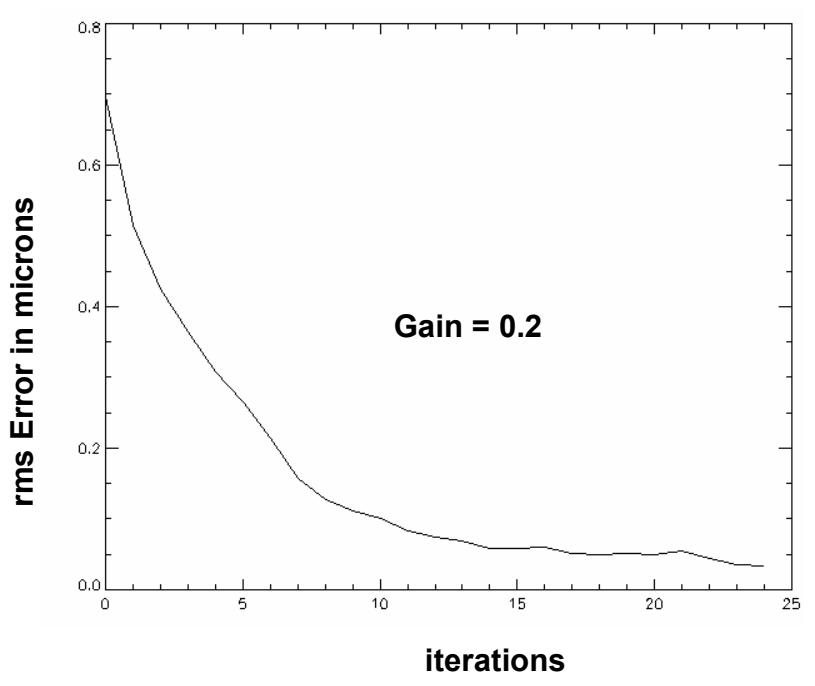

Fig. 22 Convergence of the $.25 \mathrm{D}$ lens to 0.05 microns

\section{HUMAN SUBJECTS TESTING}

In order to measure and correct aberrations of human eye, each subject must have a standard ophthalmic eye exam. During the testing period, the subjects are seated, have their eyes dilated, and bite down on a molded plastic "bite bar" to stabilize the motion of their head. This plastic bite bar is attached to a steel bar that can be adjusted to a comfortable position for the patient. To measure the aberrations of the eye, it is 
imperative that the head be still, so once in place the steel bar is tightened down, and the motion of the patients head is minimized.

The first step is to use the subjects prescription in the conventional phoropter placed in front of the eye and correct the focus and astigmatism error. Then, a flip-in mirror is inserted after the SLM, is flipped up, and the pupil of the subject is aligned (using $x-y-z$ positioner) on the pupil viewing camera to a predetermined position. Then, the mirror is removed and the subject is asked to fixate at a target on the monitor. The head position may again have to be adjusted to align the subject's pupil to the S-H sensor, if it is not already aligned. After the subject is stabilized and all the Hartmann spots are obtained, the subject is asked to maintain eye fixation at a fixed target, while the loop is being closed. Fig. 24 shows a typical Hartmann spot obtained from a human eye. The wavefront before and after correction is shown in Fig. 25. As the loop converges, the rms error reduces from $1.5 \mu$ to $0.7 \mu$ as shown in Fig. 26.

Once the vision is corrected, and the laser beam is turned off, the subject is asked to look at the computer screen and press specified buttons depending on what they see. The test is repeated a number of times to reduce the statistical variation across different measurements. These psychophysical tests are designed to provide a number corresponding to the subject's visual performance.

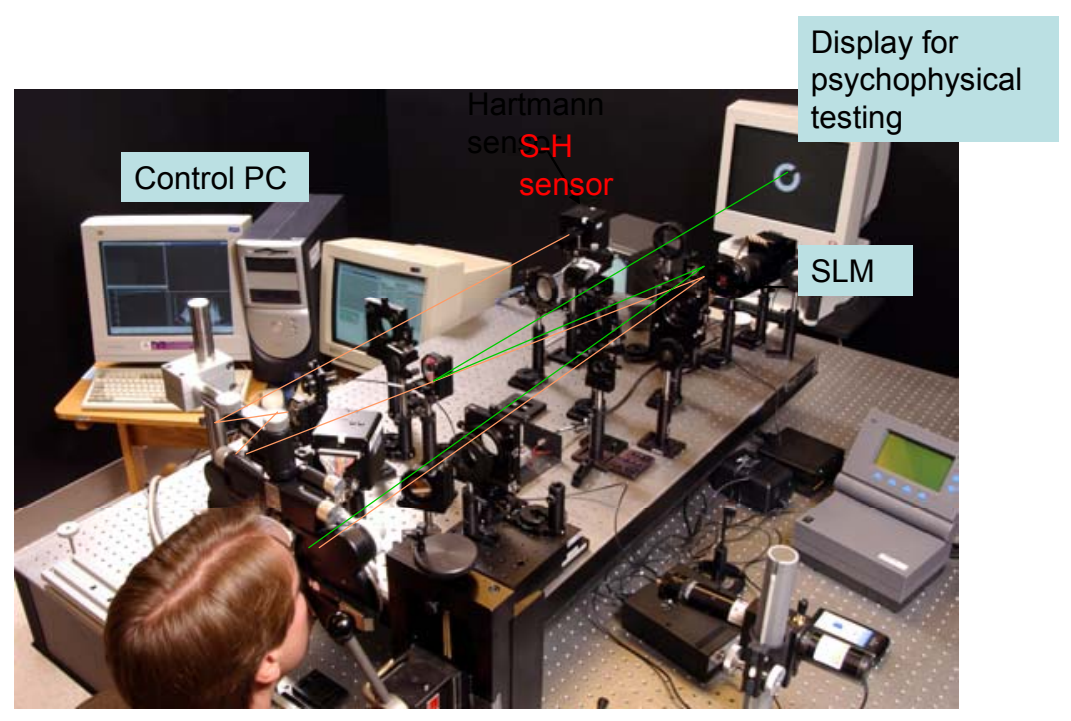

Fig. 23 Complete view of the set up 

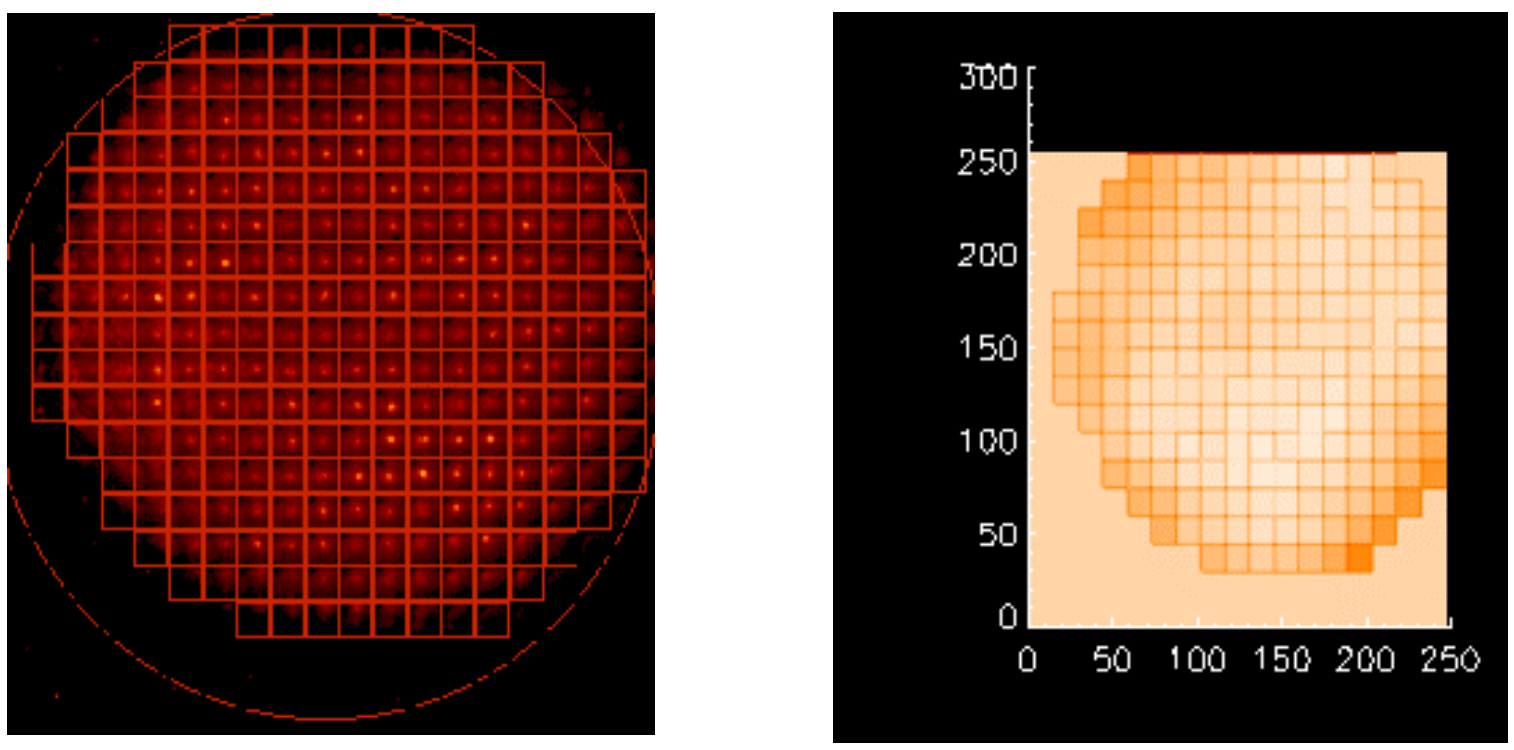

Fig. 24 A typical H-S sensor data and reconstruction $\mathrm{rms}=3.5$

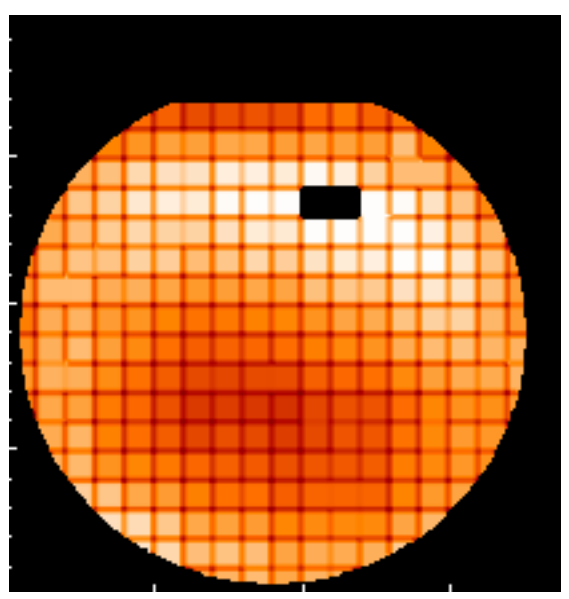

Before correction

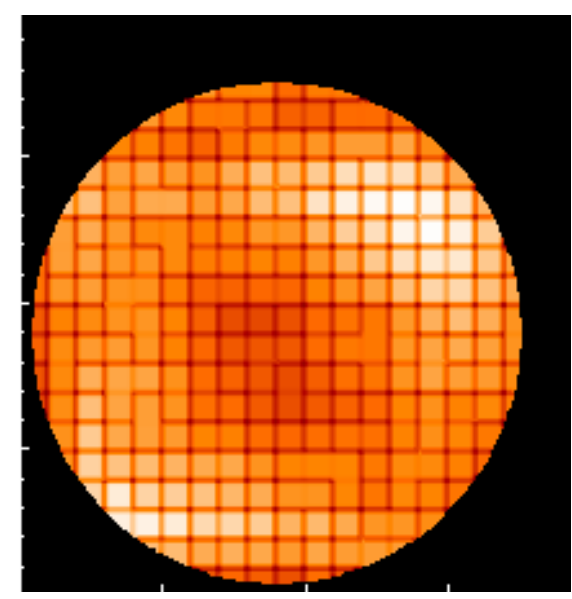

After correction

Fig. 25. Wavefronts before and after correction for a human subject 


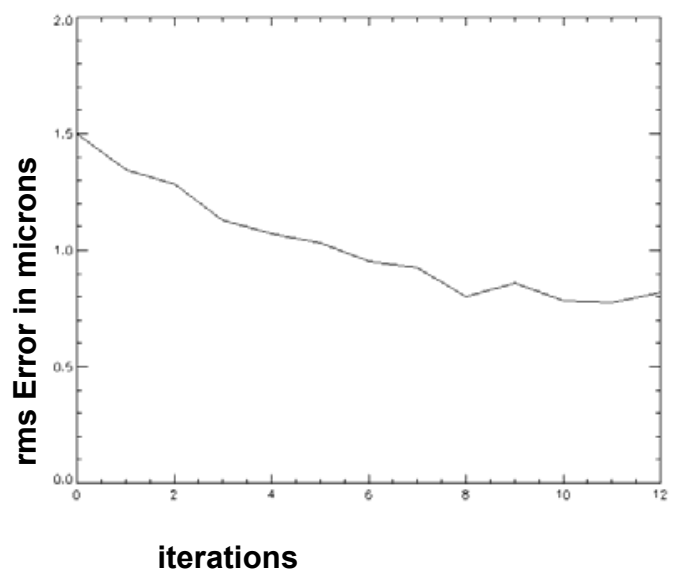

Fig. 26 Convergence of the rms error of the human eye to 0.78 microns

\section{SUMMARY}

A systematic study was undertaken to characterize the system from a systems engineering point of view. A series of steps were taken to fully characterize the performance of each the subsystems. These steps are necessary to estimate the accuracy and limitations of the system, devise necessary remedy and ensure repeatability of the measurements. These are recording the noise statistics, verifying the accuracy of the centroid calculation, performing accurate registration, understanding the effect of misregistration, characterizing the nonlinear behavior of the SLM, determining the phase wrapping and tackle the nonlinear response of the SLM using a lookup table, quantifying the performance of the wavefront sensor, verifying the close loop operation using an internally and externally generated aberration. After the above steps we were able to apply the system to human vision correction and perform psychophysical experiments. Once high order aberration correction is performed using the high resolution liquid crystal SLM, it is possible to modify the AO system to perform retinal imaging.

\section{Acknowledgement:}

This work was supported by a LDRD grant from Lawrence Livermore National Laboratory. This work was performed under the auspices of the U.S. Department of Energy by the University of California, Lawrence Livermore National Laboratory under contract No. W-7405-Eng-48. 


\section{REFERENCES}

1. C. Max et al., "Design, Layout, and early results of a feasibility experiment for sodium layer guide start adaptive optics," J. Opt. Soc. AM. A, vol. 11, pp.813824 (1994).

2. J. W. Hardy, Adaptive Optics for Astronomical telescopes, New York, Oxford University Press, 1998.

3. R. K. Tyson, Adaptive Optics, Academic, San Diego, California, 1991.

4. J. Liang, B. Grimm, S. Goelz, and J. Bille, Objective measurement of the wave aberrations of the human eye with the use of a Hartmann-Shack wave-front sensor, J. Opt. Soc. Am. A, 11, 1949-1957 (1994)

5. J. Liang, D. Williams and D. Miller, "Supernormal vision and high resolution retinal imaging through adaptive Optcs," J. Opt. Soc. Am. A, 14, 2884-2892 (1997)

6. M.W. Kartz, S.S. Olivier, K. Avicola, J. Brase, C. Carrano, D. Silva, D. Pennington, and C. Brown, "High Resolution Wavefront control of high power laser systems," 16-21, Proc. of $2^{\text {nd }}$ International workshop on Adaptive Optics for Industry and Medicine, University of Durham, England, 1999.

7. S. C. Wilks et al. "High Resolution Adaptive Optics Test-bed for Vision Science," Adaptive Optics System and Technology II, R. K. Tyson, D. Bonaccini, M.C. Roggemann Editors, Proc. Of SPIE, Vol. 4494, 349-355, 2002.

8. L.A. Poyneer, D.T. Gavel and J.M. Brase "Fast wavefront reconstruction in large adaptive optics systems with use of the Fourier transform," J. Opt. Soc. Am. (A), 19, 2100-11, (2002). 